\title{
Geographic variation in access among adults with kidney disease: evidence from medical expenditure panel survey, 2002-2011
}

\author{
Mukoso N. Ozieh ${ }^{1,2}$, Kinfe G. Bishu ${ }^{1,2}$, Rebekah J. Walker ${ }^{1,2,3}$, Jennifer A. Campbell ${ }^{1,2}$ and Leonard E. Egede ${ }^{1,2,3^{*}}$
}

\begin{abstract}
Background: To understand geographic variation in access to care over time in patients with kidney disease.

Methods: We analyzed 4404 (weighted sample of 4,251,129) adults with kidney disease from the United States using the Medical Expenditure Panel Survey over 10 years. Three dependent variables were created to investigate variation in access: usual source of care, overall medical access to care, which took into account usual source of care, ability to get care, and delay in care, and prescription access, which took into account ability to get prescriptions and delay in getting prescriptions. Multiple logistic regression was used with geographic region as the main independent variable, adjusting for relevant covariates.

Results: Compared to the Northeast region, adults living in the Midwest ( $\mathrm{OR}=0.56 ; 95 \% \mathrm{Cl}$ 0.35-0.89), South ( $\mathrm{OR}=0$. $48 ; 95 \% \mathrm{Cl} 0.32-0.72)$ and West $(\mathrm{OR}=0.53 ; 95 \% \mathrm{Cl} 0.34-0.84$ ) had significantly lower odds of reporting usual source of care. For the combined access measure, compared to Northeast, adults in Midwest (OR=0.60; $95 \% \mathrm{Cl} 0.40-0.88$ ), South $(\mathrm{OR}=0.62 ; 95 \% \mathrm{Cl} 0.44-0.88)$ and West $(\mathrm{OR}=0.50 ; 95 \% \mathrm{Cl} 0.34-0.72)$ had significantly lower odds of medical access to care. Region was not significantly associated with the odds of having prescription access, though a significant increase in prescription access was observed over time.

Conclusions: Geographic variation in access to care among adults with kidney disease exists independent of income, education, insurance and comorbid conditions, with those in the South least likely to have a usual source of care and those in the West least likely to have overall access to care when compared to the Northeast United States.
\end{abstract}

Keywords: Geographic variation, Kidney disease, Access to care, MEPS

\section{Background}

A fundamental link between healthcare systems and the populations they serve is access to care, which is a multidimensional concept concerned with determining whether those who need care can enter the healthcare system [1-3]. The Institute of Medicine defined access as "a timely use of personal health services to achieve the best possible health outcomes" [4]. While actual access can be gauged by health care utilization information, measures gathering information on the ability to receive care when it is needed may be more appropriate

\footnotetext{
* Correspondence: egedel@musc.edu

${ }^{1}$ Center for Health Disparities Research, Department of Medicine, Medical University of South Carolina, Charleston, SC, USA

${ }^{2}$ Department of Medicine, Division of General Internal Medicine and Geriatrics, Medical University of South Carolina, Charleston, SC, USA Full list of author information is available at the end of the article
}

$[2,3]$. As a result, global measures of access, such as having a primary care provider, ability to obtain care when needed, or delay in needed medical care, help evaluate overall access to care over time [3].

Studies have identified barriers to care to include financial, psychological, social, organizational, spatial, and temporal factors; and differences by social characteristics and enabling resources may indicate inequitable care $[3,5]$. Investigations note inequity in access for low-income and minority populations including irregular source of care, lack of preventative care, and delay in obtaining needed care $[3,4]$. However, fewer studies investigate regional differences in care. As region is an immutable personal factor in healthcare, variations in this measure offer insight on system-level factors that may need targeted interventions [2]. An analysis using 
data from the Medical Expenditure Panel Survey (MEPS) found regional differences in care for the general population. Americans in the West and South were less likely to have a usual source of health care than those in the Northeast and Midwest [1]. It also revealed that those in the Northeast were the least likely to encounter difficulty or delay in obtaining medical care [1]. Large regional variations were also seen in the proportion of Americans that report having a regular doctor and those diagnosed with a chronic disease that report visiting their doctors in the past 2 years [6]. As regional accessibility involves social dimensions of access beyond constraints due to distance or travel, it is important to investigate and better understand differences in access to care [7].

Chronic medical diseases, such as kidney disease, are particularly important to consider in an analysis of access to care because those with a usual source of care are more likely to obtain treatment for chronic health conditions [1]. However, though those disabled with end-stage renal disease (ESRD) receive coverage through Medicare, disparities in outcomes and access to quality care continue [8]. For instance, socioeconomic and or geographic variation has been reported in Medicare spending for people with ESRD and in the kidney transplant population, and racial/ethnic minorities have a greater burden and worse outcomes for chronic kidney disease (CKD) [9-13]. Eliminating barriers to care for those with kidney disease or risk factors for kidney disease continues to be a concern. Specifically, there is a need to provide access to care in order to monitor pre-dialysis care and prevent progression of disease $[8,14,15]$. Studies have found differences in access by poverty status, incidence and awareness of disease, and variation in Medicaid coverage; however, most studies focus on understanding disparities in kidney transplantation rather than kidney disease $[8,10,16-18]$. As a result, the association between geographic variation and access to care over time in patents with kidney disease remains unclear. To our knowledge, no study has used national data over a prolonged time period to examine geographic variation in access among adults with kidney disease.

Therefore, the aim of this study is to understand geographic variation in access to care over time in patients with kidney disease. In an effort to understand changes over time and inform policy decisions, this analysis uses global definitions for access, broadens the disease classification beyond only CKD or ESRD, and uses national data to allow for generalizable estimates.

\section{Methods}

\section{Data source and sample}

We analyzed the responses of 4404 adults ( $\geq 18$ years) with kidney disease from the United States using the
Medical Expenditure Panel Survey (MEPS) for the years 2002-2011. MEPS is a nationally representative survey of the U.S. civilian non-institutionalized population and is administered by the Agency for Healthcare Research and Quality [19, 20]. MEPS obtains comprehensive information on participants' use of medical care, prescription medication and their medical spending, as well as information on demographics, socioeconomics and satisfaction with health care. The complex survey design includes multistage sampling, clustering and stratification with oversampling of minorities [21]. Using weights provided by the Agency for Healthcare Research and Quality to account for sampling, the weighted sample for this analysis represented 4,251,129 adults with kidney disease living in the United States.

Self-reported information is collected from respondents, in addition to collection of data on medical and financial variables from all types of health care providers for validation and supplementation [19]. Diagnosis coded according to International Classification of Disease, Ninth Revision, Clinical Modification (ICD-9-CM) are also collected. Kidney disease related medical conditions and procedures reported by respondents were recorded by an interviewer as verbatim and then converted by professional coders to ICD-9-CM codes. Fully specified ICD-9$\mathrm{CM}$ codes were collapsed into three digits in order to protect confidentiality of respondents [20]. The error rate for any coder did not exceed $2.5 \%$ on verification [22]. For each year, data were merged from the medical condition files and the full-year consolidated files using the unique person identifier (DUPERSID) on a one-to-one match [20]. To ensure sufficient sample size and robust estimation for our analysis [23-25], we pooled the 10-year MEPS data.

\section{Measures}

All measures are based on previously validated questionnaires that are publicly available on the MEPS website $[19,20]$. Individuals with kidney disease were identified from the MEPS household medical condition files using clinical classification categories (CCCs) codes of 156 (nephritis, nephrosis, renal sclerosis), 157 (acute and unspecified renal failure), 158 (chronic renal failure), 160 (calculus of urinary tract) and 161 (other diseases of kidney and ureters) [20].

The dependent variables in this study were dichotomized variables created from questions asked in the access section of MEPS. The first access variable was Usual Source of Care, as determined by response to the question: Do you have a usual source of care provider? An overall Medical Access to Care variable was created from responses to three separate questions: 1) Do you have a usual source of care provider?, 2) Were you unable to get necessary medical care?, and 3) Were you 
delayed in getting necessary medical care?. If respondents answered 'yes' to having a usual source of care, 'no' to being unable to get necessary care, and 'no' to having a delay in necessary medical care they were coded as having Medical Access. Opposite answers to any of the three questions resulted in being coded as not having Medical Access. An overall Prescription Access to Care variable was created from responses to two questions: 1 ) Were you unable to get necessary prescription medication?, and 2) Were you delayed in getting necessary prescription medication? [20]. Similarly to Medical Access, if respondents answered 'no' to being unable to get prescriptions, and 'no' to having a delay in necessary prescriptions they were coded as having Prescription Access. Opposite answers to either of the two questions resulted in being coded as not having Prescription Access.

The primary independent variable was census region coded as: Northeast, Midwest, South and West.

\section{Covariates}

All covariates used for analysis were based on self-report and were included to take into account sociodemographic differences between the regions. Binary indicators of co-morbidities were based on a positive response to a question "Have you ever been diagnosed with...?". Cardiovascular disease (CVD), however, was a positive response to diagnosis with coronary heart disease, angina, myocardial infarction, or other heart diseases. Race/ethnic groups are categorized as: Non-Hispanic White (NHW), Non-Hispanic Black (NHB), Hispanic or others. Education was categorized as: less than high school ( $\leq$ grade 11), high school (grade 12) and college or more (grade $\geq 13$ ). Marital status was coded as: married, non-married (widowed/divorced/separated) and never married. Gender was dichotomized and age was coded into three age groups: $18-44,45-64$ and $\geq$ 65 years. Metropolitan Statistical Areas (MSA) was dichotomized based on population as of end of the year. Health insurance was coded as: private, public only and uninsured at all time in the year. The income level was defined as a percentage of the poverty level and grouped in to four categories: poor ( $<125 \%$ of poverty level), low income (125\% to less than $200 \%$ of poverty level), middle income (200\% to less than $400 \%$ of poverty level) and high income $(\geq 400 \%$ of poverty level). Calendar year was grouped into 2002/03, 2004/05, 2006/07, 2008/ $09,2010 / 11$ for the pooled data.

\section{Statistical analysis}

We used multiple logistic regressions with binary variables of Usual Source of Care, Medical Access to Care, and Prescription Access to Care as the dependent variables (yes versus no) across geographic region, adjusting for age, sex, race/ethnicity, marital status, education, insurance status, MSA status, household income, comorbidities and calendar year. For interpretation, we use the adjusted odds ratio coefficient of the logistic regression.

F-adjusted mean residual goodness-of-fit was applied to test the adequacy of the models. After fitting the logistic regression models taking the survey design in to account, the F-adjusted mean residual goodness-of-fit suggested no evidence of lack of fit [26]. The link test that account complex survey design, used as a diagnostic test to examine the model specification error, verified no evidence of model specification error in the models [27]. Using the Variance inflation factor (VIF) test, and taking into account the complex survey design, it was determined that no multicollinearity problems existed between predictors of the models. All analyses were performed at the person-level using STATA 14 (StataCorp LP College Station, TX). Only estimates that are statistically significant at the $p<0.05$ level are discussed.

\section{Results}

Characteristics of U.S. Adults with kidney diseases (KD)

Characteristics of adults with kidney diseases (KD) in the study are summarized in Table 1 . Of the sample population representing 4404 U.S. adults with KD, $15.5 \%$ were from Northeast, $19.7 \%$ were from Midwest, $42.5 \%$ were from South and the remaining $22.3 \%$ were from the West region. Non- Hispanic Whites with KD were more likely in the Midwest region (83.2 compared to $73.6 \%$ mean), Non-Hispanic Blacks with KD were more likely in the South region (14.4 compared to $11.3 \%$ mean), and Hispanic and Others with KD were more likely in the West region (22.9 compared to $11.1 \%$ mean for Hispanics, and 9.1 compared to $4.0 \%$ mean for Other) $(p<0.001)$. Privately insured adults with KD were least likely in the West (57.4 compared to 64.4\% mean), whereas publicly insured adults with KD were more likely in the West (33.3 compared to $28.2 \%$ mean), and uninsured adults with KD were more likely in the South and West regions (9.3 for both compared to $7.4 \%$ mean) $(p<0.001)$. Poor and low income individuals with $\mathrm{KD}$ were more likely in the Midwest region (22.4 compared to $19.6 \%$ mean), while high income individuals with KD were more likely in the Northeast region (41.3 compared to $36.3 \%$ mean) $(p<0.001)$.

\section{Access to care}

Table 2 presents the results from the logistic regression of whether adults with KD indicated usual source of care. In Table 3, we report our findings from logistic regression of whether adults with KD had medical access to care, as defined in the methods. Table 4 shows the logistic regression of whether adults with $\mathrm{KD}$ had prescription access. 
Table 1 Sample demographics by geographical region among U.S. adults with kidney disease (KD)

\begin{tabular}{|c|c|c|c|c|c|c|}
\hline Variables & All (\%) & Northeast (\%) & Midwest (\%) & South (\%) & West (\%) & $P$-value \\
\hline$N(n)$ & $4,251,128(4404)$ & 797,487 (683) & $880,779(869)$ & $1,746,049(1870)$ & $826,813(982)$ & \\
\hline \multicolumn{7}{|l|}{ Age category } \\
\hline Age $18-44$ & 29.6 & $29.7^{\prime}$ & 30.1 & 29.7 & 29.0 & \multirow[t]{3}{*}{0.799} \\
\hline Age $45-64$ & 36.6 & 38.7 & 37.5 & 36.0 & 34.5 & \\
\hline Age $65-85$ & 33.8 & 31.6 & 32.4 & 34.3 & 36.5 & \\
\hline \multicolumn{7}{|l|}{ Gender } \\
\hline Male & 51.2 & 49.8 & 52.8 & 51.2 & 50.9 & \multirow[t]{2}{*}{0.885} \\
\hline Female & 48.8 & 50.2 & 47.2 & 48.8 & 49.1 & \\
\hline \multicolumn{7}{|l|}{ Race/ethnicity } \\
\hline Non-Hispanic White & 73.6 & 75.9 & 83.2 & 73.0 & 62.5 & \multirow[t]{4}{*}{$<0.001$} \\
\hline Non-Hispanic Black & 11.3 & 9.7 & 11.8 & 14.4 & 5.5 & \\
\hline Hispanic & 11.1 & 10.6 & 3.1 & 9.8 & 22.9 & \\
\hline Other & 4.0 & 3.8 & 1.9 & 2.8 & 9.1 & \\
\hline \multicolumn{7}{|l|}{ Marital status } \\
\hline Married & 56.9 & 57.4 & 60.5 & 55.1 & 56.5 & \multirow[t]{3}{*}{0.254} \\
\hline Non-married ${ }^{a}$ & 28.6 & 25.0 & 27.0 & 30.7 & 29.5 & \\
\hline Never married & 14.5 & 17.6 & 12.5 & 14.2 & 14.0 & \\
\hline \multicolumn{7}{|l|}{ Education category } \\
\hline$<$ High school & 21.9 & 17.8 & 20.4 & 24.2 & 22.7 & \multirow[t]{3}{*}{0.011} \\
\hline High school & 33.1 & 40.3 & 35.1 & 30.3 & 29.8 & \\
\hline College or more & 45.0 & 41.9 & 44.5 & 45.5 & 47.5 & \\
\hline \multicolumn{7}{|l|}{ Insurance } \\
\hline Private & 64.4 & 68.9 & 67.5 & 64.0 & 57.4 & \multirow[t]{3}{*}{$<0.001$} \\
\hline Public & 28.2 & 26.1 & 28.3 & 26.7 & 33.3 & \\
\hline Uninsured & 7.4 & 5.0 & 4.2 & 9.3 & 9.3 & \\
\hline \multicolumn{7}{|c|}{ Metropolitan statistical status } \\
\hline MSA & 81.3 & 86.3 & 73.1 & 81.1 & 85.6 & \multirow[t]{2}{*}{0.014} \\
\hline Non-MSA & 18.7 & 13.7 & 26.9 & 18.9 & 14.4 & \\
\hline \multicolumn{7}{|l|}{ Poverty category } \\
\hline Poor/NEA & 19.6 & 18.5 & 16.9 & 20.3 & 22.4 & \multirow[t]{4}{*}{$<0.001$} \\
\hline Low Income & 15.5 & 14.1 & 17.7 & 15.1 & 15.2 & \\
\hline Middle Income & 28.6 & 26.1 & 29.6 & 29.0 & 28.8 & \\
\hline High Income & 36.3 & 41.3 & 35.8 & 35.6 & 33.6 & \\
\hline \multicolumn{7}{|l|}{ Chronic conditions } \\
\hline Diabetes & 28.9 & 25.6 & 28.3 & 29.6 & 31.5 & \\
\hline Hypertension & 56.1 & 52.2 & 56.2 & 56.6 & 59.0 & 0.347 \\
\hline CVD & 30.4 & 28.3 & 29.9 & 30.6 & 32.3 & 0.679 \\
\hline Stroke & 8.4 & 7.9 & 8.3 & 7.8 & 10.1 & 0.642 \\
\hline Emphysema & 4.1 & 3.2 & 3.2 & 4.7 & 4.3 & 0.457 \\
\hline Joint pain & 51.0 & 44.6 & 51.9 & 52.8 & 52.6 & 0.022 \\
\hline Arthritis & 41.9 & 39.9 & 44.1 & 43.5 & 38.2 & 0.162 \\
\hline Asthma & 11.5 & 11.0 & 10.0 & 11.9 & 12.8 & 0.521 \\
\hline \multicolumn{7}{|l|}{ Year category } \\
\hline Year 2002/03 & 17.7 & 18.4 & 18.9 & 17.6 & 16.0 & 0.901 \\
\hline
\end{tabular}


Table 1 Sample demographics by geographical region among U.S. adults with kidney disease (KD) (Continued)

\begin{tabular}{|c|c|c|c|c|c|c|}
\hline Year 2004/05 & 18.4 & 17.3 & 19.6 & 18.5 & 17.8 & \\
\hline Year 2006/07 & 19.3 & 20.9 & 19.1 & 18.6 & 19.5 & \\
\hline Year 2008/09 & 20.9 & 22.2 & 20.6 & 20.0 & 22.0 & \\
\hline Year 2010/11 & 23.7 & 21.2 & 21.8 & 25.3 & 24.7 & \\
\hline \multicolumn{7}{|l|}{ Access } \\
\hline Usual source of care & 89.7 & 93.4 & 90.6 & 88.1 & 88.8 & 0.004 \\
\hline Medical access & 82.1 & 88.1 & 82.6 & 81.2 & 77.9 & $<0.001$ \\
\hline Prescription access & 91.8 & 94.4 & 92.2 & 90.8 & 90.8 & 0.064 \\
\hline
\end{tabular}

$\mathrm{N}$-weighted sample size; $\mathrm{n}$-unweighted sample size; \%, weighted percentage

${ }^{a}$ Non-married stands for widowed/divorced and separated

Compared to the Northeast region, adults living in the Midwest $(\mathrm{OR}=0.56 ; 95 \% \mathrm{CI} 0.35-0.89)$, South $(\mathrm{OR}=0.48 ; 95 \% \mathrm{CI} 0.32-0.72)$ and West $(\mathrm{OR}=0.53$; $95 \%$ CI 0.34-0.84) had significantly lower odds of reporting a usual source of care. Other covariates significantly associated with usual source of care included higher age, (aged 45-64 $(\mathrm{OR}=2.0 ; 95 \% \mathrm{CI}$ $1.48-2.85)$ and aged $65-85(\mathrm{OR}=2.95 ; 95 \% \mathrm{CI} 1.96-$ 4.43), female gender ( $\mathrm{OR}=1.43$; $95 \% \mathrm{CI} 1.10-1.87)$. Covariates significantly associated with less usual source of care included uninsured ( $\mathrm{OR}=0.28$; 95 \% CI 0.20 0.40). Comorbid conditions like diabetes $(\mathrm{OR}=1.47 ; 95 \%$ CI 1.02-2.12), hypertension (OR-2.08; 95 \% CI 1.532.81) and CVD (OR $=1.56 ; 95 \%$ CI 1.09-2.21) had a higher odds but emphysema had a lower odds $(\mathrm{OR}=0.37$; $95 \%$ CI 0.16-0.86) of having usual source of care. Compared to the bench mark year of 2002/03, calendar year 2008/09 had a lower odds of having a usual source of care $(\mathrm{OR}=0.65 ; 95 \%$ CI 0.43-0.97).

When considering the combined access measure, compared to Northeast region, adults in Midwest $(\mathrm{OR}=0.60$; 95 \% CI 0.40-0.88), South (OR $=0.62$; 95 \% CI 0.44$0.88)$ and West $(\mathrm{OR}=0.50 ; 95 \%$ CI $0.34-0.72)$ had significantly lower odds of medical access to care. Again more access was associated with age (aged 45-64 (OR = 1.80; 95 \% CI.39-2.32) and aged 65-85 (OR-2.70; $95 \%$ CI 1.91 - 3.82)), and female gender ( $\mathrm{OR}=1.22$; $95 \% \mathrm{CI}$ 1.0-1.48). Other race/ethnicity had a lower odds of medical access $(\mathrm{OR}=0.55 ; 95 \%$ CI $0.35-0.85)$ compared with their non-Hispanic white counterparts, as did uninsured adults $(\mathrm{OR}=0.22 ; 95 \%$ CI $0.16-0.30)$ compared with privately insured groups. Higher income groups had statistically significant higher odds of medical access $(\mathrm{OR}=1.46 ; 95 \% \mathrm{CI}$ 1.05-2.03). Adults with comorbid hypertension had higher odds of medical access $(\mathrm{OR}=1.52$; $95 \% \mathrm{CI} 1.20-1.93)$, but joint pain had a lower odds $(\mathrm{OR}=0.75 ; 95 \%$ CI $0.59-0.94)$ compared to their counter parts.

Region was not significantly associated with the odds of having prescription access, and compared to 2002/03, both calendar years (2006/07 and 2008/09) were associated with higher odds of prescription access.

\section{Discussion}

The results of this study demonstrate geographic variation in access to care in patients with kidney disease over time, with a significant decrease in having a usual source of care in 2008/09 compared to 2002/03. Access to prescriptions showed no geographic variation, however, there was a significant increase in access to prescription medications across the years investigated. Amongst adults with kidney disease, living in the South, West, and Midwest is associated with a lower likelihood of usual source of care and lower overall access to care compared to living in the Northeast. Additionally, adults with kidney disease with comorbid diabetes, hypertension, and CVD had 1.5 to 2 times higher likelihood of usual source of care whereas those with comorbid emphysema had a lower likelihood of usual source of care.

To our knowledge, this study is the first of its kind to investigate trends in geographical variation in access to care over a decade using a nationally representative sample among adults with kidney disease. While prior research has established geographic variation in access to care among patients with ESRD and kidney transplantation $[10,16,17]$, this study demonstrates disparate access to care for patients with any diagnosis of kidney disease. This suggests that poor outcomes for those with CKD and ESRD may be linked to barriers to access occurring early in the disease process. Lack of regular access to care may serve to compound disease burden and result in the early onset of disease related complications. For instance, as reported by the US Renal Data System, nearly half of the patients who started receiving treatment for CKD never received care from a nephrologist prior to CKD onset [28]. While barriers to care are multidimensional and can be influenced at the patient level by disease awareness and patient preference; the geographic variation in access, as well as outcomes, suggest that system level barriers to access may have an 
Table 2 Logistic regression model for usual source of care among adults with kidney disease (KD) by geographical status

\begin{tabular}{llll}
\hline Variables & Odds ratio & $95 \% \mathrm{Cl}$ & $p$ value \\
\hline Primary independent variables &
\end{tabular}

Northeast (ref)
Midwest
South
West
Covariates
Age
Age 18-44 (ref)
Age 45-64
Age 65-85
Gender

Female
Race/ethnicity

Non-Hispanic White (ref)

Non-Hispanic Black

Hispanic

Others

Marital Status

Married (ref)

Not married ${ }^{a}$

Never married

Education

$<$ High school (ref)

High school

College or more

nsurance Status

Private (ref)

Public insured

Uninsured

MSA status

MSA

Poverty Category

Poor/NEA (ref)

Low Income

Middle Income

High Income

Chronic Conditions ${ }^{b}$

Diabetes

Hypertension

CVD

Stroke

Emphysema

Joint Pain
0.92

0.71

0.65

0.86

0.82

$0.64^{*}$

$0.61^{* *}$

0.45-0.93

0.44.- -0.84

0.019

0.003

$0.56^{*}$

0.35-0.89

$0.34-0.84$

0.58-1.48

0.49-1.02

$0.38-1.11$

-
0.016

0.001

0.007

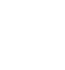

$-$

$<0.001$

$<0.001$

0.008

$-$

0.753

0.071

0.119

$-$

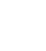

\subsection{0-1.22 0.403}

0.58-1.15 $\quad 0.262$

$-$

0.909

$<0.001$

$0.28^{* * *}$

$0.70-1.49$

$0.20-0.40$

$0.79-1.55$

0.544

$0.74-1.72$

$0.93-1.92$

$0.94-2.18$

$1.02-2.12$

$1.53-2.81$

$1.09-2.21$

$0.49-2.23$

$0.16-0.86$

$0.83-1.51$
$0.32-0.72$
Table 2 Logistic regression model for usual source of care among adults with kidney disease (KD) by geographical status (Continued)

\begin{tabular}{llll}
\hline Arthritis & 1.38 & $0.97-1.97$ & 0.071 \\
Asthma & 1.35 & $0.89-2.05$ & 0.148 \\
Year & & & \\
Year 2002/03 (ref) & - & - & - \\
Year 2004/05 & 0.93 & $0.62-1.41$ & 0.766 \\
Year 2006/07 & 0.72 & $0.48-1.08$ & 0.117 \\
Year 2008/09 & $0.65^{*}$ & $0.43-0.97$ & 0.039 \\
Year 2010/11 & 0.80 & $0.52-1.22$ & 0.306 \\
\hline
\end{tabular}

*Level of significance $p<0.05$; ${ }^{* *}$ level of significance $p<0.01$, ${ }^{* *}$ level of significance $p<0.001$

${ }^{a}$ Non-married stands for widowed/divorced and separated

${ }^{\mathrm{b}}$ Reference for each chronic conditions was not having that specific condition

impact on disease complications and outcomes through a patient's ability to obtain care.

In this analysis we investigated three related, but conceptually separate measures of access to care: having a usual source of care, a more comprehensive measure of access incorporating considerations including delay of care, and having access to prescriptions. As access is a complex construct, it is important to consider all three to have a better understanding of overall access to care for patients with kidney disease. These results suggest that while there are no geographic differences in access to prescriptions, differences do exist in access to medical care, whether using a more basic measure such as usual source of care, or a more comprehensive measure. In addition, while in both medical access measures, those with kidney disease in the Northeast were more likely to have access, the relative differences between other regions and the Northeast differed between the two measures. While those in the South had the lowest odds of having a usual source of care, it was those in the West that had the lowest odd of having access when taking ability to obtain care, and not having a delay in care into account. These results support findings of a commonwealth fund report that revealed geography influences access to care and quality of care [29]. Similar to our study, they found that areas in the Northeast and Midwest had higher measures of access compared to areas in the South. They also found strong relationships between access to care and outcomes, with better access being associated with better outcomes. In the Southeast region, it has also been reported that testing and detection effort are low compared to other regions [30]. Unfortunately, this at-risk region (the Southeast) has the highest prevalence of recognized CKD, further suggesting the need to examine the impact that system level barriers may have in the variation in access for patients with kidney disease and those at risk for CKD and ESRD. 
Table 3 Logistic regression model for medical access to care among adults with kidney disease (KD) by geographical status

\begin{tabular}{llll}
\hline Variables & Odds ratio & $95 \% \mathrm{Cl}$ & $p$ value \\
\hline Primary independent variables &
\end{tabular}

Northeast (ref)
Midwest
South
West
Covariates
Age
Age 18-44 (ref)
Age 45-64
Age 65-85
Gender

Female
Race/ethnicity

Non-Hispanic White (ref)

Non-Hispanic Black

Hispanic

Others

Marital Status

Married (ref)

Not married ${ }^{a}$

Never married

Education

$<$ High school (ref)

High school

College or more

Insurance Status

Private (ref)

Public insured

Uninsured

MSA status

MSA

Poverty Category

Poor/NEA (ref)

Low Income

Middle Income

High Income

Chronic Conditions ${ }^{b}$

Diabetes

Hypertension

CVD

Stroke

Emphysema

Joint Pain

\section{$0.60^{* *}$ \\ 0.40-0.88}

$0.62^{* *}$

$0.50^{* * *}$

$0.44-0.88$

0.34-0.72

0.009

0.008

$<0.001$

(2)

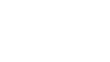

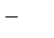

$1.80^{* * *}$

$2.70^{* * *}$

$1.22^{*}$

1.10

0.99

$0.55^{* *}$

$$
-
$$

\subsection{9-2.32}

$1.91-3.82$

$1.0-1.48$

.

$-$

$<0.001$

$<0.001$

0.048

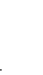

$-$

$0.65^{* *}$

$0.62^{* *}$

0.50-0.85

0.47-0.83

0.002

0.001

$-$

$$
0.72-1.26
$$$$
0.68-1.21
$$

0.769

0.542

0.578

0.975

0.009

$-$

0.02

001

.769
.542

$-$

0.754

$<0.001$

$0.16-0.30$

$0.76-1.33$

0.945

0.78-1.56

0.547

1.11

1.25

$1.46^{*}$

0.93-1.68

0.124

$1.05-2.03$

0.024

$0.74-1.34 \quad 0.749$

$1.20-1.93<0.001$

$0.85-1.38 \quad 0.510$

$0.59-0.94 \quad 0.437$

$0.39-1.26 \quad 0.241$

$\begin{array}{lll}0.70 & 0.39-1.26 & 0.241 \\ 0.75^{*} & 0.59-0.94 & 0.014\end{array}$

Table 3 Logistic regression model for medical access to care among adults with kidney disease (KD) by geographical status (Continued)

\begin{tabular}{llll}
\hline Arthritis & 1.19 & $0.92-1.54$ & 0.166 \\
Asthma & 0.92 & $0.67-1.26$ & 0.626 \\
Year & & & \\
Year 2002/03 (ref) & - & - & - \\
Year 2004/05 & 0.99 & $0.70-1.40$ & 0.975 \\
Year 2006/07 & 0.93 & $0.66-1.32$ & 0.715 \\
Year 2008/09 & 0.95 & $0.68-1.31$ & 0.770 \\
Year 2010/11 & 0.88 & $0.63-1.24$ & 0.491 \\
\hline
\end{tabular}

*Level of significance $p<0.05$; **level of significance $p<0.01,{ }^{* * *}$ level of significance $p<0.001$

${ }^{a}$ Non-married stands for widowed/divorced and separated

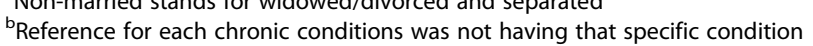

The Healthy People 2020 objective for chronic kidney disease (CKD) is to reduce new cases of CKD and its complications, disability, death and economic costs [31]. Access to care ensures at-risk individuals get timely, preventative and recommended care so as to obviate when possible the complications and economic burden of kidney disease. Lack of access to care not only affects an individual's ability to maintain their health, but also negatively impacts quality of life as a whole. Therefore, understanding geographic variation in access is vital for establishing successful policies geared towards reducing the incidence, prevalence and complications of kidney disease across the nation [30]. The Affordable Care Act (ACA) is one of the most recent national undertakings to improve access to care. The ACA-Medicaid expansion was implemented as part of this act to improve access for low income Americans, but this mandate is dependent on state implementation [32]. Unfortunately, many of the states that have chosen not to implement this law are in at-risk regions of the USA based on this analysis, such as the Southeast [33]. This study serves as baseline information for policymakers on existing regional variation in access to care among adults with kidney disease in the midst of the Affordable Care Act (ACA) reform. Future studies should consider how the ACA-Medicaid expansion influenced variations seen.

\section{Conclusions}

The strengths of this study include the use of nationally representative data over a prolonged period, including broad care categories (access to usual source of care, medical access care, and prescription access care) and the use of a robust estimation method to examine geographic variation in access to care accounting for comorbidities among patents with kidney disease. However, limitations do exist. First, MEPS relies on self-report, and as such may be prone to potential bias and recall 
Table 4 Logistic regression for prescription access among adults with kidney disease (KD) by geographical status

\begin{tabular}{llll}
\hline variables & Odds ratio & $95 \% \mathrm{Cl}$ & $p$ value \\
\hline
\end{tabular}

Primary independent variables

Northeast (ref)

Midwest

South

West

Covariates

Age

Age 18-44 (ref)

Age 45-64

Age 65-85

Gender

Female

Race/ethnicity

Non-Hispanic White (ref)

Non-Hispanic Black

Hispanic

Others

Marital Status

Married (ref)

Not married ${ }^{a}$

Never married

Education

$<$ High school (ref)

High school

College or more

nsurance Status

Private (ref)

Public insured

Uninsured

MSA status

MSA

Poverty Category

Poor/NEA (ref)

Low Income

Middle Income

High Income

Chronic Conditions ${ }^{b}$

Diabetes

Hypertension

CVD

Stroke

Emphysema

Joint Pain
0.77

0.68

0.64

$0.57^{* *}$

0.84

$0.39-0.82$

0.54-1.29

0.003

0.436

-

0.284

0.051

0.064

$\sqrt{2}+x^{2}$

$-$

0.310

$<0.001$

0.278

$-$

0.463

0.049

0.934

-

0.209

0.059

$0.98-2.09$

$-$

0.164

$<0.001$

$0.23-0.56$

$0.66-1.36$

0.807

0.95

1.14

1.14

1.22

$0.67^{*}$

0.72

0.80

1.06

0.88

$0.55^{* * *}$
Table 4 Logistic regression for prescription access among adults with kidney disease (KD) by geographical status (Continued)

\begin{tabular}{llll}
\hline Arthritis & 1.05 & $0.75-1.45$ & 0.759 \\
Asthma & $0.51^{* *}$ & $0.35-0.75$ & 0.001 \\
Year & & & \\
Year 2002/03 (ref) & - & - & - \\
Year 2004/05 & 1.30 & $0.87-1.94$ & 0.193 \\
Year 2006/07 & $1.65^{*}$ & $1.08-2.52$ & 0.019 \\
Year 2008/09 & $2.02^{* *}$ & $1.32-3.10$ & 0.001 \\
Year 2010/11 & 1.50 & $0.96-2.35$ & 0.074 \\
\hline
\end{tabular}

*Level of significance $p<0.05$; ${ }^{* *}$ level of significance $p<0.01$, ${ }^{* *}$ level of significance $p<0.001$

${ }^{a}$ Non-married stands for widowed/divorced and separated

${ }^{\mathrm{b}}$ Reference for each chronic conditions was not having that specific condition

error. However, it is one of the few national dataset with extensive information on health care access, utilization and cost, and questions have been validated over time to collect reliable information. Second, there is no laboratory information in MEPS and the ICD-9 codes for chronic kidney disease are collapsed for confidentiality reasons, so we are unable to examine geographic trends by CKD stage. Third, while the panel design of this study and the ability to investigate responses over different years provides trends over time, individual respondents were not followed over time, and therefore the results of our study should not be interpreted longitudinally.

This study provides insight into geographic variation in access to care among adults with kidney disease independent of income, education, insurance and comorbid conditions. Compared to the Northeast, living in the South, West, and Midwest is associated with lower likelihood of having a usual source of care or having overall access to care. Conversely, no geographic variation was seen in access to prescription medications. These findings are the first of its kind to examine trends in access in adults with kidney disease and serves as baseline for further studies evaluating the impact of potential policy reforms to access.

Acknowledgements

Not applicable.

Funding

This study was supported by the National Institute of Diabetes and Digestive and Kidney Diseases (grant K24DK093699, Principal Investigator: Leonard Egede, MD).

Availability of data and materials

The datasets generated and analyzed during the current study are available in the Agency for Healthcare Research and Quality (AHRQc). Medical Expenditure Panel Survey. 2011 Full year consolidated data file 2013c, Available from http:// meps.ahra.gov/mepsweb/data_stats/download_data_files.jsp. 


\section{Authors' contributions}

$\mathrm{MO}$ was a major contributor in writing the manuscript. KB analyzed and interpreted the data, and contributed to drafting the article. JC was a major contributor in writing the manuscript. RW and LE were contributors to drafting the article and revised the article critically for important intellectual content. All authors were involved in conception and design and approved the final manuscript

\section{Competing interests}

The authors declare that they have no competing interests.

\section{Consent for publication}

Approval to publish is provided for publicly available MEPS data.

\section{Ethics approval and consent to participate}

This analysis used secondary data from the Medical Expenditures Panel Survey, as such all ethics approvals and consent to participate was waived.

\section{Author details}

${ }^{1}$ Center for Health Disparities Research, Department of Medicine, Medical University of South Carolina, Charleston, SC, USA. ${ }^{2}$ Department of Medicine, Division of General Internal Medicine and Geriatrics, Medical University of South Carolina, Charleston, SC, USA. ${ }^{3}$ Health Equity and Rural Outreach Innovation Center (HEROIC), Ralph H. Johnson Veterans Affairs Medical Center, Charleston, SC, USA.

Received: 27 July 2016 Accepted: 12 October 2016

Published online: 18 October 2016

\section{References}

1. Weinick RM, Drilea SK. Usual sources of health care and barriers to care, 1996. StatBull Metrop Insur Co. 1998;79(1):11-7.

2. Aday LA and Andersen RM. A framework for the study of access to medical care. Health Services Research. 1974;9(3):208-20.

3. Andersen RM, Davidson PL. Improving access to care in America: Individual and contextual indicators. In Changing the US Health Care System: Key Issues in Health Services Policy and Management. $3^{\text {rd }}$ edition. Eds: Andersen RM, Davidson PL, Baumeister SE. San Francisco. Jossey-Bass. 2007. Pages 3-31.

4. Institute of Medicine (US). Committee on Monitoring Access to Personal Health Care Services. Access to Health Care in America. Ed: M. Millman. Washington DC: National Academy Press. 1993.

5. Donabedian A. Models for organizing the delivery of personal health services and criteria for evaluating them. Milbank Mem Fund Q. 1972;50:103.

6. Radley DC, Schoen C. Geographic variation in access to care - the relationship with quality. N Engl J Med. 2012;367(1):3-6.

7. Comber AJ, Brunsdon C, Radburn R. A spatial analysis of variations in health access: linking geography, socio-economic status and access perceptions. Int J Health Geogr. 2011;10:44.

8. Williams AW. Health policy, disparities, and the kidney. Adv Chronic Kidney Dis. 2015;22(1):54-9.

9. Axelrod DA, Lentine $\mathrm{KL}$, Xiao $\mathrm{H}$, et al. Accountability for end-stage organ care: implications of geographic variation in access to kidney transplantation. Surgery. 2014;155(5):734-42.

10. Mathur AK, Ashby VB, Sands RL, Wolfe RA. Geographic variation in endstage renal disease incidence and access to deceased donor kidney transplantation. Am J Transplant. 2010;10(4 Pt 2):1069-80.

11. Francis A, Didsbury M, Lim WH, et al. The impact of socioeconomic status and geographic remoteness on access to pre-emptive kidney transplantation and transplant outcomes among children. Pediatr Nephrol. 2016;31(6):1011-19.

12. Hirth RA, Tedeschi PJ, Wheeler JR. Extent and sources of geographic variation in Medicare end-stage renal disease expenditures. Am J Kidney Dis. 2001;38(4):824-31.

13. Garcia-Garcia G, Jha V. World Kidney Day Steering C. Chronic kidney disease in disadvantaged populations. Transplantation. 2015;99(1):13-6.

14. Crews DC, Greer RC, Fadrowski JJ, et al. Setting an agenda for comparative effectiveness systematic reviews in CKD care. BMC Nephrol. 2012;13:74

15. London R, Solis A, Goldberg GA, Wade S, Chan WW. Examination of resource use and clinical interventions associated with chronic kidney disease in a managed care population. J Manag Care Pharm. 2003;9(3):248-55.
16. Mohan S, Mutell R, Patzer RE, Holt J, Cohen D, McClellan W. Kidney transplantation and the intensity of poverty in the contiguous United States. Transplantation. 2014;98(6):640-5.

17. Patzer RE, Pastan SO. Kidney transplant access in the Southeast: view from the bottom. Am J Transplant. 2014;14(7):1499-505.

18. Shah A, Fried LF, Chen SC, et al. Associations between access to care and awareness of CKD. Am J Kidney Dis. 2012;59(3 Suppl 2):S16-23.

19. Agency for Healthcare Research and Quality (AHRQa). Methodology Report \# 27, Sample design of the 2011 Medical Expenditure Panel Survey Insurance Component 2013a, Available from http://meps.ahrq.gov/data_ files/publications/mr27/mr27.pdf. Accessed 13 Oct 2016.

20. Agency for Healthcare Research and Quality (AHRQb). Medical Expenditure Panel Survey. 2011 Full year consolidated data file 2013c, Available from http://meps.ahrq.gov/mepsweb/data_stats/download_data_files.jsp. Accessed 18 Aug 2014.

21. Agency for Healthcare Research and Quality (AHRQc). MEPS HC-036: 19962011 Pooled Linkage Variance Estimation File 2013d. MEPS file, Available from http://meps.ahrq.gov/data_stats/download_data/pufs/h36/h36u11doc. shtml. Accessed 13 Oct 2016.

22. Agency for Healthcare Research and Quality (AHRQ). Household Component- Insurance Component Linked Data 1999, Research file 2003, Available from http://meps.ahrq.gov/mepsweb/data_stats/download data/ pufs/link_99hcic/hc_ic99link_doc.pdf. Accessed 13 Oct 2016.

23. Desai PR, Lawson KA, Barner JC, Rascati KL. Identifying patent characteristics associated with high Schizophrenia-related direct medical costs in community-dwelling patents. J Manag Care Pharm. 2013;19(6):468-77.

24. Ozieh MN, Bishu KG, Dismuke CE, Egede LE. Trends of healthcare expenditure in United States in adults with diabetes: 2002-2011. Diabetes Care. 2015;38(10):1844-51.

25. Egede LE, Bishu KG, Walker RJ, Dismuke CE. Impact of diagnosed depression on healthcare costs in adults with and without diabetes: United States, 2004-2011. J Affect Disord. 2016;195:119-26.

26. Archer KJ, Lemeshow S. Goodness-of-fit test for a logistic regression model fitted using survey sample data. Stata J. 2006;6(1):97-105.

27. Musman S, Passos VMA, Silva IBR, Barreto SM. Evaluation of a prediction model for sleep apnea in patients submitted to polysomnography. J Bras Pneumol. 2011:37(1):75-84.

28. US Renal Data System. USRDS 2010 Annual Data Report: Atlas of Chronic Kidney Disease and End-Stage Renal Disease in the United States. National Institutes of Health, National Institute of Diabetes and Digestive and Kidney Diseases, Bethesda, MD, 2010.

29. D. C. Radley, S. K. H. How, A. K. Fryer, D. McCarthy, and C. Schoen, Rising to the Challenge: Results from a Scorecard on Local Health System Performance, 2012, The Commonwealth Fund, March 2012. www. commonwealthfund.org/publications/fund-reports/2012/mar/localscorecard.

30. Collins AJ, Chen SC, Gilbertson DT, Foley RN. CKD surveillance using administrative data: impact on the health care system. Am J Kidney Dis. 2009;53(3 Suppl 3):S27-36

31. Healthy People 2020 https://www.healthypeople.gov/. Accessed 13 Oct 2016

32. Sommers BD, Maylone B, Nguyen KH, Blendon RJ, Epstein AM. The Impact Of State Policies On ACA Applications And Enrollment Among Low-Income Adults In Arkansas, Kentucky, And Texas. Health Aff (Millwood). 2015 Jun;34 (6):1010-8.

33. State Reforum http://www.nashp.org/states-stand-medicaid-expansiondecisions/. Accessed 23 Apr 2016. 